\title{
Transverse dural arteriovenous fistula with bilateral sigmoid sinus stenosis treated with via transarterial embolization and venous stent placement: case report
}

\section{Zeran Yu}

the First Affiliated Hospital of Kunming Medical University

\section{Hai Song}

the First Affiliated Hospital of Kunming Medical University

Jiang Long ( $\sim 374524085 @ q q . c o m$ )

the First Affiliated Hospital of Kunming Medical University

\section{Yiqiang Cao}

the First Affiliated Hospital of Kunming Medical University

\section{Yonggang Wang}

the First Affiliated Hospital of Kunming Medical University

\section{Xiang Yang}

the First Affiliated Hospital of Kunming Medical University

\section{Case report}

Keywords: dural arteriovenous fistula, bilateral sinus stenosis, intracranial hypertension, embolization, stenting

Posted Date: June 23rd, 2021

DOI: https://doi.org/10.21203/rs.3.rs-575847/v1

License: (c) (i) This work is licensed under a Creative Commons Attribution 4.0 International License. Read Full License 


\section{Abstract}

\section{Background}

Dural arteriovenous fistulas (DVAF) is a cerebrovascular disorder due to abnormal connections within meningeal arteries and cerebral veins. It is usually associated with sinus stenosis.

\section{Case Presentation}

We describe a case of DAVF with bilateral sigmoid sinus stenosis successfully treated via transarterial embolization and unilateral stenting.

\section{Conclusion}

The author reported a rare case of DAVF associated with with bilateral sigmoid sinus stenosis. Direct embolization with catheterization of contralateral MMA may by an alternative way to treat partial embolized DAVF, when main feeding arteries were occluded due to previous treatment. Unilateral sinus recanalization for bilateral sinus narrows could also obtain obvious benefit.

\section{Introduction}

Dural arteriovenous fistulas (DAVF) is a pathological connection between intracranial arteries and cerebral veins resulting from trauma, previous skull surgery or intracranial venous thrombosis pathology of dAVF[1 3]. For patients with both DVAF and sinus stenosis, interventional embolization and sinus stenting could achieve good results $[4,5]$.For complicated DAVF requiring staged embolization, however, superlative embolization routines could be occluded due to previous treatment[6, 7]. Moreover, bilateral sinus stenosis are challenging[5]. We present an atypical case of DAVF with bilateral sinus occlusion successfully treated by contralateral feeder artery embolization and unilateral sinus recanalization.

\section{Case Report}

History and Examination

A 38-year-old woman complaining left eye blindness, right eye blurred vision and headache for one year was referred to our department. Ophthalmofundoscope demonstrated papilledema indicating intracranial hypertension. The opening pressure of lumbar puncture was $400 \mathrm{mmH}_{2} \mathrm{O}$ in local hospital. Headache could be released by administration of mannitol. Four months later, due to constant headache and deteriorating eyesight, she was referred local hospital and was diagnosed as right transverse dural arteriovenous fistula with bilateral sigmoid severe stenosis. Transarterial embolization occluded right middle meningeal artery and right occipital artery, but failure of recanalization of left sigmoid sinus. Follow-up cerebral angiography performed two months later demonstrated that the fistula still fed by right meningohypophyseal trunk and the branches of posterior cerebral artery, and bilateral sigmoid sinus were severe stenosis. Recanalization of left sinus was still failed in local hospital. 
Angiographic findings:

Digital subtraction angiography demonstrated both intracranial and extracranial circulations supplying the DAVF. Contrast injection in the right internal carotid artery (ICA) revealed filling of the DAVF from the meningohypophyseal trunk (Fig. 1, A). Contrast injection in the right external carotid artery (ECA) demonstrated filling of the DAVF form the occipital artery. Contrast injection in the left ECA revealed filling of the DAVF from the middle meningeal artery and occipital artery (Fig. 1, C, D). Contrast injection in the right vertebral artery revealed filling of the DAVF from right posterior meningeal artery and right superior cerebellar artery. Contrast injection in the left vertebral artery revealed filling of the DAVF from parietooccipital artery of left posterior cerebral artery and left posterior meningeal artery. There is no blood supply the fistula from the branches of left ICA. Venous drainage into superior sagittal sinus and transverse sinus with retrograde flow into cortical veins (Fig. 1, A, D). Cortical vein engorgement and bilateral sigmoid sinus significant stenosis ( $\mathbb{9} 90 \%$ ) were observed (Fig. 1, B,C).

Operation

The patient and his family provided written informed consent for the procedure, which was performed under general anesthesia. The right femoral vein was accessed using an 8-Fr long sheath, and an 8-Fr guiding catheter (Brite Tip straight guiding catheter, Cordis, Johnson \& Johnson) was introduced over a hydrophilic 0.035 guidewire and was navigated into the right internal jugular vein at the level of internal jugular foramen of skull base. A 6-Fr diagnostic catheter was placed in the left ICA to guide contrast injections. Microcatheter (Echelon 10, ev3) was introduced over a 0.014 microwire (Synchro 14, Stryker) get through the stenosis and into the superior sagittal sinus. Microcatheter contrast injection demonstrated retrograde venous blood flow into superior sagittal sinus and straight sinus. An angioplasty balloon $(6 \times 40 \mathrm{~mm})$ was dilated at the stenotic segment of the junction between left transverse sinus and sigmoid sinus. A self-expending stent $(6 \times 40 \mathrm{~mm})$ was deployed at the terminal portion of transverse sinus. Contrast injection in the left ICA demonstrated that retrograde flow in sinus markedly decreased. When prepared for transarterial DAVF embolization, right posterior meningeal artery, right superior cerebellar artery and right meningohypophyseal trunk were not accessible due to previous embolization. Therefore, we chose embolizing DAVF via contralateral middle meningeal artery. Microcatheter (Echelon 10, ev3) was introduced over a 0.014 mirowire (Synchro 14, Stryker) into the left middle meningeal artery closed to the fistulous point. Oxyn-18 (eV3) was injected under blank fluoroscopic roadmap. Special attention was paid to maintain a gradual and progressive injection rate, while looking for any reflux of the embolizing agent that would signal to pause the injection. Contrast injection in bilateral ICA and ECA demonstrated that right meningohypophyseal trunk fed the fistula, but there is no blood supply from bilateral middle meningeal arteries, bilateral posterior meningeal arteries and right superior cerebellar artery.

\section{Postoperative Course}

The patient was administrated subcutaneously low-molecular-weight heparin enoxaparin $(20 \mathrm{mg} / 0.2 \mathrm{ml})$ twice a day for 5 days and orally warfarin $2.5 \mathrm{mg}$ once a day. She was discharged on the 5 th 
postoperative day and was placed on $2.5 \mathrm{mg}$ of warfarin daily monitoring INR between 2 to 3 at local hospital. At the 3-month telephone follow-up, the patient said the symptoms of headache had obviously minimized.

\section{Discussion}

Cases of patients with DAVF and sinus stenosis have been literately reported before[ $1,3,6,8]$. Multiple factors are believed involving the development of DAVF, but their true etiology is still unidentified Sinus thrombosis is deemed to be the most common reason for venous hypertension and ultimately dAVF formation, but the cause-effect relationship is still under investigation $[9,10]$. We believed that the DAVF with bilateral sigmoid sinus stenosis in this patient would be caused by idiopathic intracranial hypertension. Bateman et al. first proposed that a disordered positive biofeedback mechanism may be present in idiopathic intracranial hypertension where elevated CSF pressure compresses the venous sinuses, leading to further venous hypertension and further elevation of CSF pressure[11]. Abnormal high venous pressure against vessel walls established pathological connection between dural arteries and cerebral veins, resulting in DAVF appearance. Meanwhile, venous hypertension could cause venous blood congestion resulting in local tissue hypoxia, which promotes angiogenesis by releasing angiogenic factors $[1,12]$.

Almost $66 \%$ of type lla + b DAVF could cause intracranial hypertension[13], but bilateral venous congestion caused by transverse sinus stenosis leads to further venous hypertension and further elevation of intracranial hypertension. As a result, the management of sinus stenosis and DAVF were of equal prominence in this case. A series of cases about DAVF with ispilateral sinus stenosis cured by stenting were reported. Renieri et al. demonstrated two patients with Cognard classification type II DAVF and ispilateral transverse sinus stenosis had improvement after deploying a stent across the stenosis to compress both the stenosis and fistula[8]. In these cases, the location of sinus stenosis were close to the opening of fistula so that deploying a stent could both recanalization of sinus and closure of the fistula opening. In our patient, however, compression and alleviation of the fistula was not achieved because the stent failed to cover the opening of DAVF. Further embolization was needed for DAVF.

Different treatment options have been proposed to address DAVF, including endovascular embolization, surgery and radiosurgery $[7,12,14]$. Directly removing the fistula, which has a high obliteration rate, is reputed to be unsafe due to the risks associated with open surgery. Although the affected left sigmoid sinus was obliterated, incidental surgical damage of transverse sinus would cause irreversible impairment. Transvenous embolization of DAVF have been reported to be successful in the region of cavernous sinus or transverse sinus[12,14]. However, this approach was not feasible in this case considering it was difficult for a microcatheter to pass through the stent and it was possible that Onyx reflux could cause occlude the sinus. Optimal arterial routines, right middle meningeal artery, have been occluded in previous transarterial embolization. Therefore, we decided to embolize the fistula through contralateral middle meningeal artery. The rate of success is far lower after previously failed embolization due to occlusion of main feeder. However, fistula could still be embolized in a sole approach 
considering the primary benefit of Onyx is the ability to perform a slow, large-volume, controlled injection[15, 16]. Large amount of Onyx can result in filling of the fistulous network and allow for reflux into either intracranial or extracranial feeding arteries in a single catheterizations and embolization. Cognard[17] et al. reported 18 cases of DAVF were completely obliterated after embolization of only one feeder in only one-time administration. Although contralateral middle meningeal artery was distal to the lesion and its diameter is smaller compared with left middle meningeal artery and right occipital artery, cured fistula was almost achieved via this routine.

We also found the evaluation results of sigmoid sinus stenosis between cerebral angiography and magnetic resonance venography (MRV) were inconsistence. In venous stage, cerebral digital subtraction angiography (DSA) demonstrated engorgement of cortical veins with filling defects in bilateral sigmoid sinus. In magnetic resonance venography (MRV), however, images showed poor signal intensity in right transverse-sigmoid sinus, but segmented filling defect in left transverse sinus. The differences between DSA and MRV could be caused by relevant insufficient injection volume of contrast in DSA because of overflow of cortical veins and relevant inadequate filling of transverse-sigmoid sinus. It is a technical dilemma of whether nor not increasing injection volume of contrast in patients with both DAVF and sinus stenosis considering the potential risk of hemorrhage when overload injection[11,18]. This is no trials concerning the optimal parameters of contrast media injector when performed in patients with DAVF and sinus stenosis, so further studies are needed. In addition, the disagreements about the stenosis also were problematic when we were choosing stenting in left or right side sinus. Based on the result of MRV, the segmented stenosis in left transverse sigmoid seemed to be easier for recanalization, but previous two efforts proved that the sinus of left side was not accessible in this patient. Considering prior performances, surprising success of opening right side remind us it is worth the effort to try on other side if opening one side was difficult.

\section{Conclusion}

Direct embolization with catheterization of contralateral MMA may by an alternative way to treat partial embolized DAVF, when main feeding arteries were occluded due to previous treatment. Unilateral sinus recanalization for bilateral sinus narrows could also obtain obvious benefit.

\section{Declarations}

\section{Ethical Approval and Consent to participate}

The institutional review board at Lifespan exempted this case report from formal review as per the Human Research Protection Program Policy and Procedure Manual, as it does not meet the Common Rule definition for research. The patient consented for the preparation and submission of this manuscript.

\section{Consent for publication}


Written consent has been obtained from the patient involved in this case report, and the patient has agreed to his clinical information being published.

\section{Availability of data and materials}

Data sharing not applicable to this article as no datasets were generated or analyzed.

\section{Competing interests}

The authors declare that they have no competing interests

\section{Funding}

Not applicable.

\section{Authors' contributions}

ZY drafted the manuscript. HS and JL contributed to clinical treatment of the case. YC, YW and JL assisted in the preparation of the manuscript and interpretation of the figures. $\mathrm{XY}$ assisted in editing the manuscript. All authors read and approved the final manuscript.

\section{Acknowledgements}

We thank the medical staff of the Department of Neurosurgery, the First Affiliated Hospital of Kunming Medical University, for their help in the treatment of the case.

\section{References}

1. Liebig T, Henkes H, Brew S, Miloslavski E, Kirsch M, Kühne D. Reconstructive treatment of dural arteriovenous fistulas of the transverse and sigmoid sinus: Transvenous angioplasty and stent deployment. Neuroradiology. 2005;47(7):543-51.

2. Chaichana KL, Coon AL, Tamargo RJ, Huang J. Dural arteriovenous fistulas: Epidemiology and clinical presentation. Neurosurg Clin N Am. 2012;23(1):7-13.

3. Takada S, Isaka F, Nakakuki T, Mitsuno Y, Kaneko T. Torcular dural arteriovenous fistula treated via stent placement and angioplasty in the affected straight and transverse sinuses: Case report. $J$ Neurosurg. 2015;122(5):1208-13.

4. Choi BJ, Lee TH, Kim CW, Choi CH. Reconstructive treatment using a stent graft for a dural arteriovenous fistula of the transverse sinus in the case of hypoplasia of the contralateral venous sinuses: technical case report. Neurosurgery. 2009;65(5):E994-6. discussion E996.

5. Chen K-W, Su I-C, Lee J-E, Liu H-M. Treatment of arteriovenous malformation with high-flow fistula and bilateral transverse-sigmoid sinuses stenosis resulting diffuse cortical vein engorgement and symptoms resembling carotid-cavernous fistula. Asian J Neurosurg. 2015;10(4):310-2. 
6. Levrier O, Métellus P, Fuentes S, Manera L, Dufour H, Donnet A, et al. Use of a self-expanding stent with balloon angioplasty in the treatment of dural arteriovenous fistulas involving the transverse and/or sigmoid sinus: functional and neuroimaging-based outcome in 10 patients. J Neurosurg. 2006;104(2):254-63.

7. Cognard C, Januel AC, Silva NA, Tall P. Endovascular Treatment of Intracranial Dural Arteriovenous Fistulas with Cortical Venous Drainage: New Management Using Onyx. American Journal of Neuroradiology. 2008;29(2):235-41.

8. Renieri L, Michelozzi C, Brinjikji W, Darcout J, Guenego A, Vukasinovic I, et al. PTA Stent of Dural Sinuses in Brain DAVF: A Report of 4 Cases. Clin Neuroradiol 2017.

9. Kiyosue H, Tanoue S, Okahara M, Yamashita M, Nagatomi H, Mori H. Recurrence of dural arteriovenous fistula in another location after selective transvenous coil embolization: Report of two cases. American Journal of Neuroradiology. 2002;23(4):689-92.

10. Mirza FA, Fraser JF. Multiple Dural and Pial Arteriovenous Fistulae in a Twenty-Four-Year-Old Woman in the Setting of Superior Sagittal Sinus Thrombosis: Case Report and Review of Literature. J Stroke Cerebrovasc Dis. 2016;25(10):e192-9.

11. Bateman GA. Arterial inflow and venous outflow in idiopathic intracranial hypertension associated with venous outflow stenoses. J Clin Neurosci. 2008;15(4):402-8.

12. Ohara N, Toyota S, Kobayashi M, Wakayama A. Superior sagittal sinus dural arteriovenous fistulas treated by stent placement for an occluded sinus and transarterial embolization. A case report. Interv Neuroradiol. 2012;18(3):333-40.

13. Elhammady MS, Ambekar S, Heros RC. Epidemiology, clinical presentation, diagnostic evaluation, and prognosis of cerebral dural arteriovenous fistulas. Handbook of Clinical Neurology. 2017;143:99.

14. Lv X, Jiang C, Li Y, et al. The limitations and risks of transarterial Onyx injections in the treatment of grade I and II DAVFs. Eur J Radiol. 2011;80(3):e385-8.

15. Natarajan SK, Ghodke B, Kim LJ, Hallam DK, Britz GW, Sekhar LN. Multimodality treatment of intracranial dural arteriovenous fistulas in the Onyx era: A single center experience. World Neurosurg. 2010;73(4):365-79.

16. Luo C-B, Chang F-C, Teng MM-H. Update of embolization of intracranial dural arteriovenous fistula. J Chin Med Assoc. 2014;77(12):610-7.

17. Santillan A, Nanaszko M, Burkhardt J-K, Patsalides A, Gobin YP, Riina HA. Endovascular management of intracranial dural arteriovenous fistulas: A review. Clin Neurol Neurosurg. 2013;115(3):241-51.

18. Serulle Y, Miller TR, Gandhi D. Dural Arteriovenous Fistulae: Imaging and Management. Neuroimaging Clin N Am. 2016;26(2):247-58.

\section{Figures}



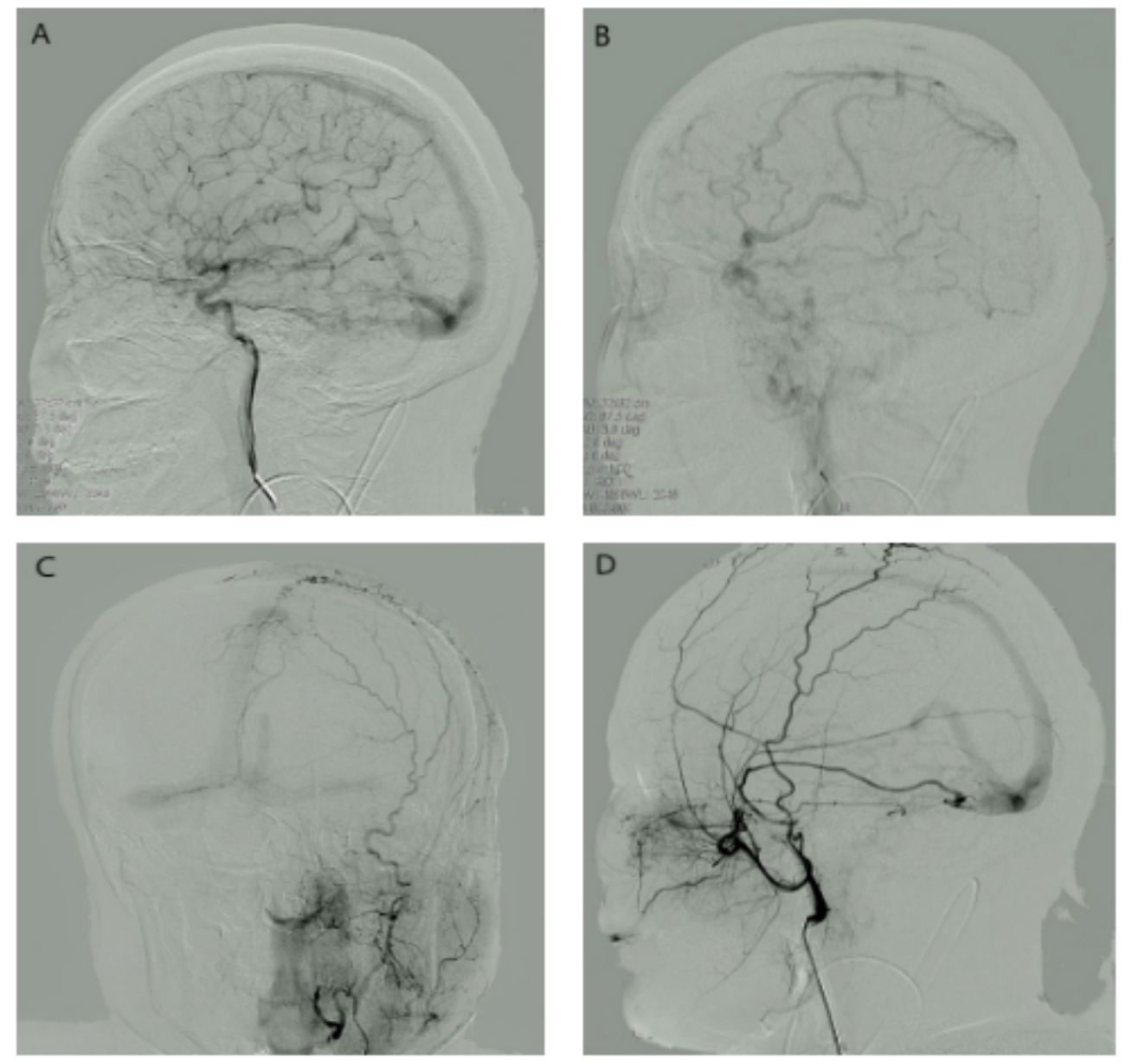

\section{Figure 1}

Cerebrovascular angiography before treatment. (A) Right meningohypophyseal trunk feeds the fistula when contrast injection in right ICA. (B) Right cortical vein engorgement and sigmoid sinus severe stenosis in venous phase during right ICA injection. DAVF of the right transverse sigmoid sinus; anteroposterior (C) and lateral (D) projections during left ECA injection. 

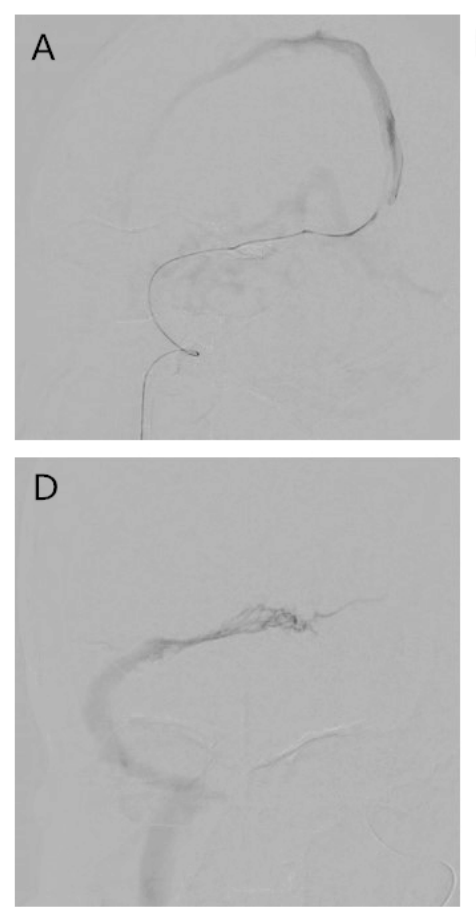
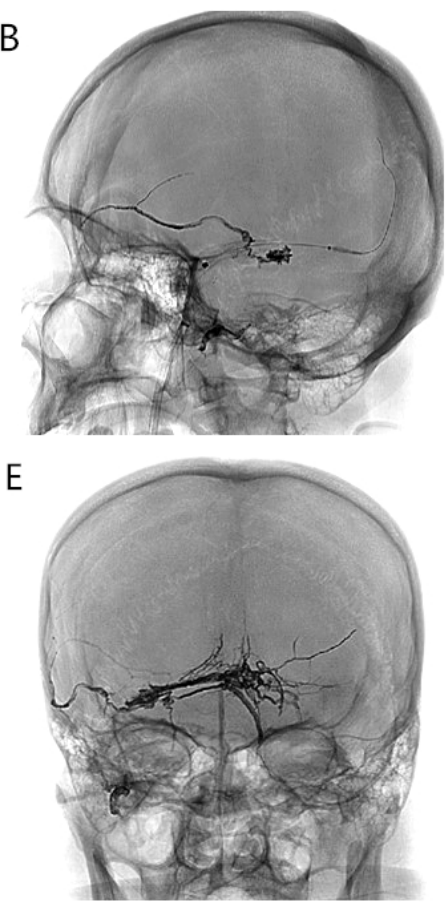

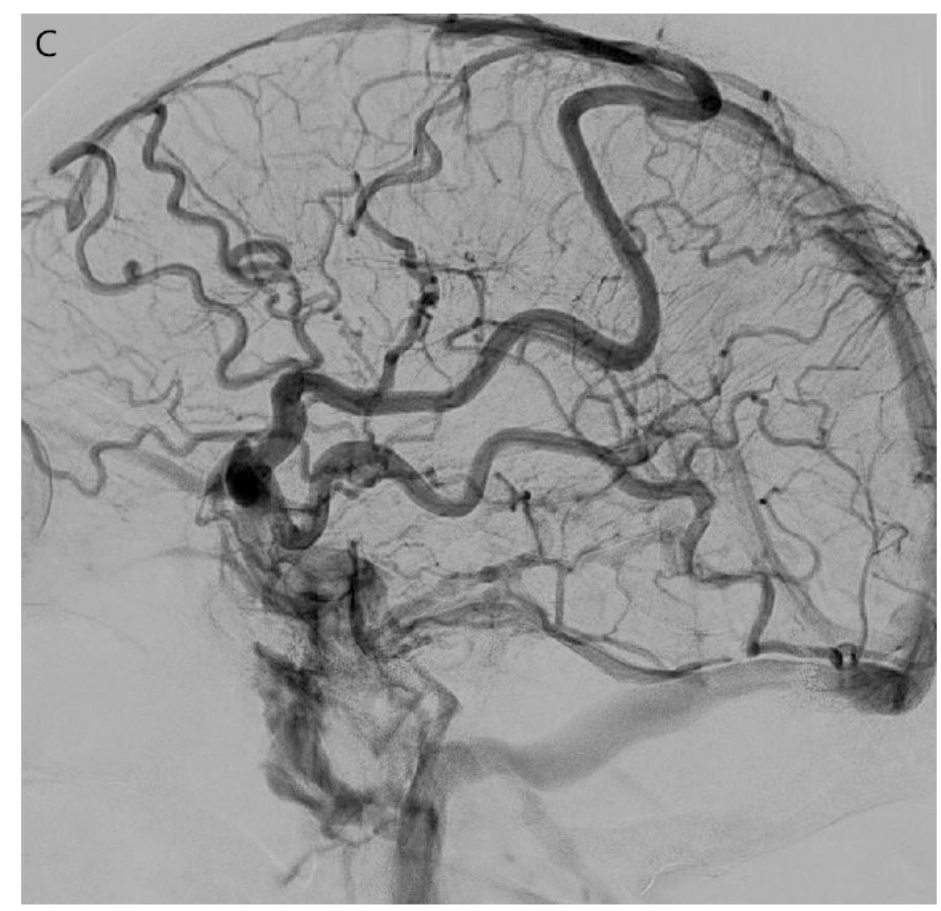

Figure 2

(A) Contrast injection from microcatheter after passing the stenosis, (B) Deployment of the stent. (C) The flow of contrast in right transverse-sigmoid sinus after angioplasty and stenting. (D) Injecting contrast from microcatheter when it is introduced to the fistula from left MMA. (E) Final cast of Onyx®
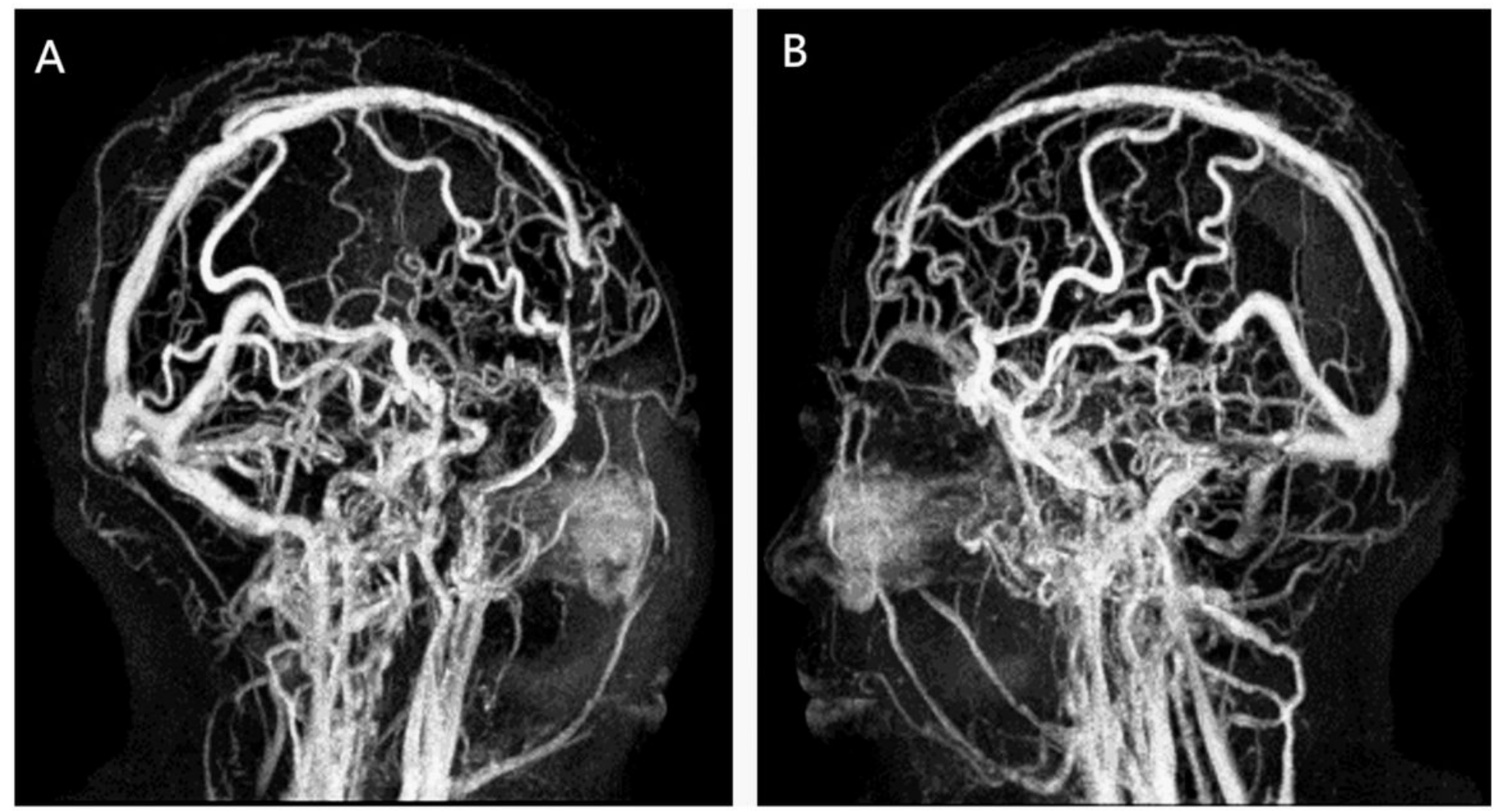

Figure 3 
MRV before treatment (A) Right transverse sinus was totally absent. (B) Left transverse sinus was segmental defects 